\title{
活细胞内微粒的跳跃运动和布朗运动
}

\author{
唐 孝 威 \\ (中国科学院高能物理研究所, 北京 100039)
}

\section{关踥调跳跃运动、布朗运动、ATP 水解}

在光学显微镜下容易看到活细胞内微粒的无规则运动以. 早期把这种无规则运动都归之 于微粒的布朗运动, 即分子热运动引起微粒的无规则运动. 但以后许多实验观察到活细胞内 微粒还发生时间上间歇、方向上无规则的跳跃运动 ${ }^{[2]}$. 跳跃运动和布朗运动这两种运动形式 容易混杂, 文献 [ 3]对区分跳跃运动和布朗运动进行过详细讨论, 但是没有定量的分析. 本文 对活细胞内微粒的跳跃运动和布朗运动进行定量讨论.

微粒的跳跃运动和布朗运动具有不同的机制. 用活细胞内 ATP 分子的化学能在短时间间 隔内转换为微粒机械能的观点, 可以解释微粒的间歇式的跳跃运动. 微粒跳跃运动是由 ATP 分子水解驱动的一种生命活动形式, 而微粒的布朗运动是由分子热运动引起的运动. 在活细 胞内的微粒, 既作布朗运动, 又可以发生跳跃运动; 但细胞外无生命的微粒只能作布朗运动而 不发生跳跃运动.

对于半径为 $a$ 的球形微粒, 我们考察它在发生跳跃运动的持续时间 $\tau$ 内的位移 $d$ 和速度 v. 设一个 ATP 分子水解提供能量 $W$, 在时间 $\tau$ 内 $n$ 个 ATP 分子水解、驱动微粒运动的能 最是 $E=n W$, 对微粒的作用力是 $F$, 细胞质粘度是 $\eta$, 根据以下关系式:

求得

$$
\begin{gathered}
d=v \tau, \\
E=F d, \\
F=6 \pi a \eta v .
\end{gathered}
$$

$$
\begin{aligned}
& d=\sqrt{\frac{E \tau}{6 \pi a \eta}}, \\
& \nu=\sqrt{\frac{E}{6 \pi a \eta \tau}} .
\end{aligned}
$$

设 $a=0.5 \mu \mathrm{m},-=10 \mathrm{~s}, n=20$, 用活细胞典型参数 $W=5 \times 10^{-20} \mathrm{~J}^{[4]}$ 和 $\eta=0.3 \times$ $0.1 \mathrm{~Pa} \cdot \mathrm{s}^{\xi]}$ 代人, 得到 $d=6 \mu \mathrm{m}, v=0.6 \mu \mathrm{m} / \mathrm{s}$. 这和文献[2]的实验数据接近.

我们再考察半径相同的球形微粒在相同时间间隔内, 在相同粘度 $\eta$ 的细胞质中的布朗运 动的位移和平均速度. 在热平衡条件下, 微粒在时间间隔 $\tau$ 内的布朗运动, 在一定轴上投影的 位移的均方根值等于 ${ }^{[0]}$ :

$$
\lambda_{\mathrm{B}}=\sqrt{\frac{k T \tau}{3 \pi a \eta}},
$$

本文 1991 年 4 月 17 日收到. 1991 年 7 月 25 日收到修改稿.

第 9 期

科学通

损 
平均速度是

$$
\frac{\lambda_{\mathrm{B}}}{\tau}=\sqrt{\frac{k T}{3 \pi a \eta r}},
$$

其中 $k$ 是波尔兹曼常数, $T$ 是绝对温度. 设 $a=0.5 \mu \mathrm{m}, \tau-10 \mathrm{~s}, \eta=0.3 \times 0.1 \mathrm{~Pa} \cdot \mathrm{s}, T=$ $293 \mathrm{~K}$, 得到 $\lambda_{B}=0.5 \mu \mathrm{m}, \frac{\lambda_{B}}{\tau}=0.05 \mu \mathrm{m} / \mathrm{s}$.

对同一个微粒说, $a$ 和 $\eta$ 是相同的. 因时间间隔 $\tau$ 值相等, 由 $d$ 和 $\lambda_{\mathrm{B}}$ 的关宗式求得

$$
\frac{d}{\lambda_{B}}-\sqrt{\frac{E}{2 k T}} \text {. }
$$

用前面有关数值代人, 估计 $\frac{d}{\lambda_{B}}=11$. 这个值和文献 [3]所给的实验数琚是相近的. 即在跳跃 运动的时间间隔内, 同一个微粒的跳跃运动的位移大于布朗运动的均方根位移. 以上讨论的 都是在时间间隔 $\tau$ 内微粒的位移.

微粒跳跃运动的特点是: 微粒在一段短时间间隔内运动, 在这段时间后, 运动停止, 表现 为停-走-停的不连续运动. 而微粒布朗运动是不停息的.连续的运动, 运动轨道是连续的, 运 动方!旬是无规的.

令比值

$$
\begin{gathered}
\alpha \equiv \frac{W}{k T}, \\
\frac{d}{\lambda_{\mathrm{B}}}=\sqrt{\frac{1}{2} n \mathrm{n} x} .
\end{gathered}
$$

式(6)可写成

在 $T=293 \mathrm{~K}$ 时, $\alpha=12$. 上面提到, 微粒的跳沜运动是生命活动的一种形式. 比值 $\alpha \gg 1$ 使得微粒跳跃运动位移显著地大于布朗运动位移. 这时, 与跳跃运动相比, 分子热运动对微粒 的冲击只是作为微扰洏存在.

下面讨论同一个细胞内部几个不同大小微粒的运动. 用 $a_{1}$ 和 $a_{2}$ 表示同一个细胞内两 个微粒的半径, $a_{1}>a_{2}$. 设两个微粒处于相同粘度的细胞质中, 第一个微粒跳跃运动位移 $d_{1}$. 与第二个微粒布朗运动均方根位移 $\lambda_{\mathrm{B} 2}$ 之比等于

$$
\frac{d_{1}}{\lambda_{\mathrm{B} 2}}=\sqrt{\frac{1}{2} n \alpha \frac{a_{2}}{a_{1}}} .
$$

因此, 在观测同一个细胞内许多微粒时, 不排除这样的可能性: 不同大小的微粓都可以发生跳 跃运动, 但大的微粒没有明显的布朗运动, 只有小的微粒作明显的布朗运动, 其位移均方根的. 大小和大的微粒跳跃运动的位移大小相当.

致谢：感谢阎隆飞教授启发性的讨论.

\section{参考文献}

[1] Kamiya, N., Protoplasmazologia 8, 3a (1959), 1.

[2] Rebhun, L. I., J. Gen. Physiol. Suppl., 50(1967), 223.

[3] Rebhun, L. 1., Intern. Rev. of Cyt., 32(1972), 93.

[4] Alberts, B. et al., Molcrular Biology of the Cell. 2nd cdition, Garland Publishing, Inc., 1989.

[5] Nicklas. R.. Ann. Ren. Biophys. Biophys. Chem., 17(1988), 431.

[6] Einstein. A., Annalen der Physik, 17(1905), 549. 